\title{
一种基于 PLC 技术的斗轮机取料稳定系统
}

A Reclaiming Stability System of Bucket Wheel Machine Based on PLC Technology

\author{
刘俊开 \\ Jun-kai Liu \\ 宁波舟山港股份有限公司北仑矿石码头分公司 中国·浙江 宁波 315800 \\ Ningbo Zhoushan Port Company Limited Beilun Ore Branch,Ningbo, Zhejiang, 315800,China
}

摘要: 斗轮机是铁矿石码头堆场上的大型机械设备。斗轮机系统可以将堆场中的物料取出到地面皮带机上去。通过 PLC 技术 的应用对斗轮机取料系统进行改造, 提高斗轮机取料流量的稳定性,降低设备的故障率与流程的稳定性。

\begin{abstract}
Bucket wheel machine is a large-scale mechanical equipment on iron ore wharf yard. The bucket wheel system can take out the materials in the yard to the ground belt conveyor. Through the application of PLC technology, the reclaiming system of bucket wheel machine is reformed to improve the stability of reclaiming flow, reduce the failure rate of equipment and the stability of process.
\end{abstract}

关键词: 斗轮机; 取料系统; PLC

Keywords : Bucker wheel; Reclaiming system; PLC

DOI : $10.36012 /$ etr.v2i5.1935

\section{1 引言}

铁矿石码头堆场堆存压力大, 对二程船出运效率要求 日益增高, 斗轮机取料作为物料出运第一个环节, 取料过程 中的瞬时流量与平均流量直接影响出场流程的每一个环节 与船舶装船效率。斗轮机是铁矿石码头上的大型机械设备, 采用一套 PLC 系统控制整个斗轮机的所有机构的运行。斗 轮机取料稳定系统是基于斗轮机原有 PLC 系统上进行开 发。

\section{2 总体思路}

铁矿石码头斗轮机取料额定流量是 $5000 \mathrm{t} / \mathrm{h}$ ，司机在取 料时会因为种种原因导致瞬时流量远超 $5000 \mathrm{t} / \mathrm{h}$ 。斗轮机取 料稳定系统首先是要将斗轮取料的瞬时流量稳定下来。

在对整个 2019 年铁矿石码头的出场流程皮带的流量进 行统计分析, 发现相对于最小瞬时流量, 最大瞬时流量才是 影响斗轮机取料波动率的主要原因, 因此斗轮机取料稳定系 统的设计主要是降低最大瞬时流量。为解决斗轮机取料流量 过大, 系统引人斗轮电动机电流反馈, 可以实时追踪瞬时流 量过大。斗轮机 PLC 为 S7 300 系列, 通过程序设计实现对流
量过大的实时控制功能。

\section{3 技术实施}

\section{1 斗轮电动机电流反馈设定}

斗轮电动机驱动方式为 ABB PSTB 系列软启动器, 与 PLC 的通讯方式为并联串行通讯, 无电动机运行状态的反馈 信号。取料稳定系统中首先增加控制线路从 PLC 到软启动 器, 采集斗轮软启动器的模拟量输出信号, 编辑软启动器可 编程输出端口, 量程按 $4 \mathrm{~mA} 20 \mathrm{~mA}$ 对应 $0 \mathrm{~mA} 800 \mathrm{~A}$ 电流。 PLC 导轨上增加模拟量输入模块 SM331 AI4x0/4to20mA 接

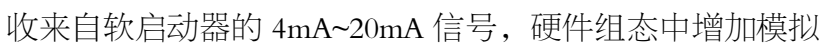
量输入模块，然后在变量表中对模拟量输入模块进行定义， 在数据块 DB401 中对斗轮软启电流数据进行定义:

\section{2 斗轮电动机电流反馈的应用}

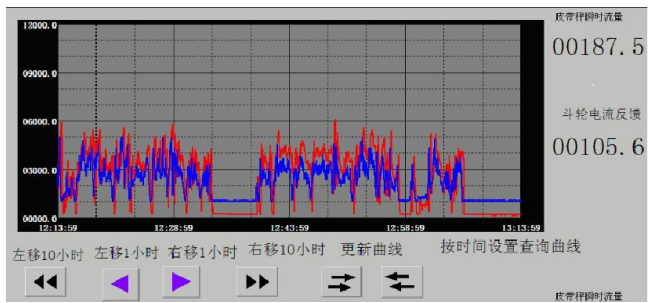

图 1 曲线查询及数据库报表

【作者简介】刘俊开(1982 ), 汉, 河北邢台, 中级职称, 港 $\square$ 装卸电气设备。 
通过 PLC 接收斗轮电流反馈信号, 转换为 REAL 数值。 在组态软件中制作历史数据趋势曲线将取自电子皮带秤的 瞬时流量与斗轮电流反馈进行对比, 设置曲线查询及数据库 报表,趋势曲线如图 1 所示。

其中灰色曲线为斗轮取料瞬时流量, 黑色曲线为斗轮电 动机电流, 从图 1 中大致可以看出黑色曲线略微提前于灰色 曲线, 从斗轮取料开始到皮带机电子秤大约有 $21 \mathrm{~m}$ 左右的 距离, 皮带机速度为 $3.15 \mathrm{~m} / \mathrm{s}$, 因此斗轮取料以后的物料通过 电子秤就是 $7 \mathrm{~s}$ 以后。通过组态软件记录历史数据然后将数 据导出到 EXCEL。

通过大量的历史数据记录分析, 发现斗轮电动机取料斗 轮与皮带机瞬时量成线性关系, 是正相关。采用线性公式将 斗轮电流折算瞬时取料流量: $\mathrm{E}=\mathrm{KI}+\mathrm{B}$ 为斗轮取料流量, 式 中 $: \mathrm{K}$ 为常数, $\mathrm{B}$ 为常数 $(\mathrm{B}$ 设为理想状态在本系统按 0 计 算)。斗轮机取料额定能力是 $5000 \mathrm{t} / \mathrm{h}$, 最大能力 $6250 \mathrm{t} / \mathrm{h}$, 作 为分段保护, 将瞬时流量进行分段标定当瞬时流量在 $2500 \mathrm{t} / \mathrm{h} \sim 5000 \mathrm{t} / \mathrm{h}$ 计算出 $\mathrm{K}_{1}=11.3$, 对应斗轮电流范围为 $221 \mathrm{~A} \sim 443 \mathrm{~A}$ 当瞬时流量在 $5000 \mathrm{t} / \mathrm{h} \sim 6250 \mathrm{t} / \mathrm{h}$ 计算出 $\mathrm{K}_{2}=10.8$, 对应电流范围为 443A 575A。

\section{3 通过 PLC 完成取料稳定系统}

在面对天气恶劣、矿种恶劣、视野有盲角、上层塌料和下 层单斗机堆料等多种情况下，司机较难及时发现取料量过 多。在前面通过数据分析得出斗轮电流与斗轮取料流量之间 的对应关系，就可以通过设计 PLC 程序来弥补人力的漏洞 尽量缩小流量过大持续时间。

斗轮机取料工艺为定点回转分层取料。定点分层取料就 是, 大车机构切入一定深度后停止不动, 俯仰机构对料堆进 行分层, 单回转机构动作进行取料。回转机构的动作快慢是 决定取料流量的关键, 回转机构为变频驱动无极调速, 由一 台变频器拖动 2 台电动机。斗轮机 PLC 的 CPU 为 S7 300 系 列, 使用的编程软件为 STEP7 V5.5。在原有系统程序内增加 稳定流量系统的内容。依据大量数据, 斗轮电流在 $443 \mathrm{~A}$ 以下
时, 斗轮取料处于额定取料能力 $5000 \mathrm{t} / \mathrm{h}$ 以下, 此时不对回转 速度进行限制。斗轮电流在 $443 \mathrm{~A} \sim 575 \mathrm{~A}$ 之内时, 斗轮取料流 量在 $5000 \mathrm{t} / \mathrm{h} \sim 6250 \mathrm{t} / \mathrm{h}$, 设备超负荷运行, 对流程系统运行稳 定性也有影响, 通过程序设定回转运行速度上限为全速的 $30 \%$ 来降低流量。斗轮电流在 $575 \mathrm{~A}$ 以上时, 斗轮机取料流量 大于 $6250 t / h$ 超过斗轮机最大取料量会对设备造成损伤造成 流程故障, 因此此时强制回转速度为 $0 \%$, 待流量降下来以后 恢复回转运行。

在 STEP7 中定义系统相关变量: T200 过流量延时设定 延时 2s, 中间继电器 M410.9 实现过流量停机。在回转给定程 序块内进行编程实现上述逻辑, 如下:

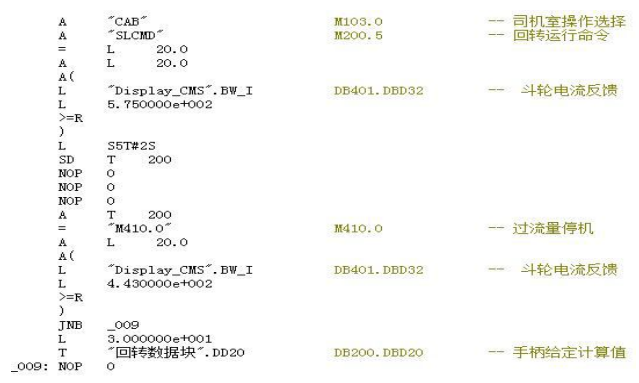

\section{4 结语}

斗轮机取料稳定系统可以有效发挥 PLC 控制系统功 能, 建立斗轮机取料运动规划, 减少斗轮机取料作业时的冲 击振动降低维修成本, 降低故障率, 提高机械使用寿命, 增加 作业可靠性, 提高斗轮机连续作业能力, 为铁矿石码头的二 程船出运提供有力的支持。 参考文献

[1] 郝坤, 李齐权, 刘立坤.斗轮式取料机瞬时取料量自动控制技术的 研究及其节能应用[J].河北工业课件,2014,(4):327-331.

[2] 吕民荟,李毅敏.斗轮堆取料机的发展趋势.港口装卸,2008(2):6-9

[3] 毛亿.斗轮堆取料机取料工艺过程分析[J].低碳世界,2016(4):203204.

[4] 吴文.港口机械设备管理[M]. 北京: 中国石油大学出版社, 2016 . P80 